EDITORA

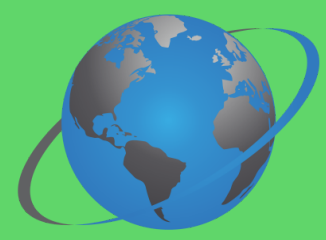

OMNIS SCIENTIA

\title{
SAÚDE PÚBLICA EM
}

\section{TEMPOS PANDEMICOS}

\section{VOLUME 1}

Organizador:

Raul Sousa Andreza

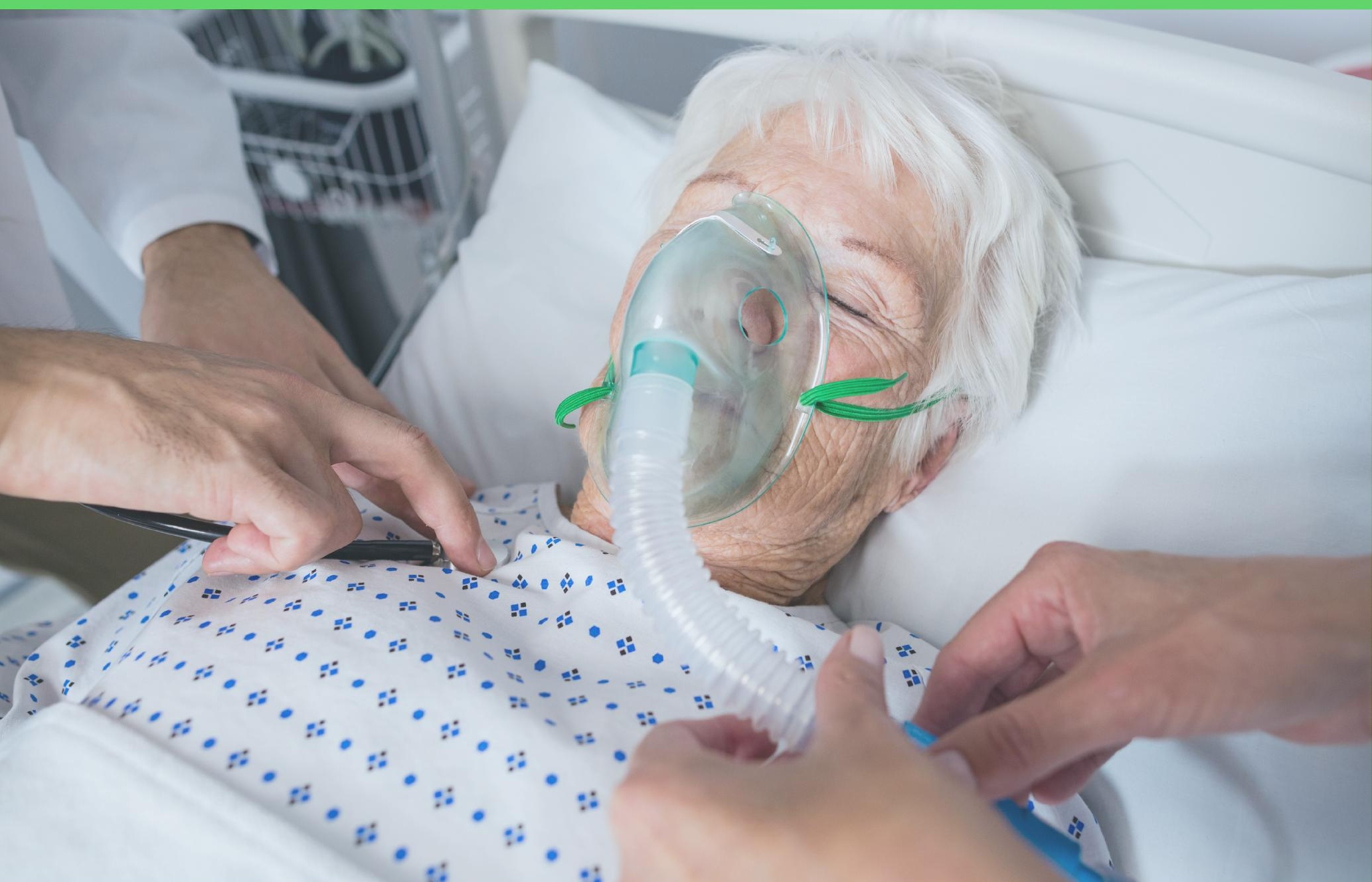


EDITORA

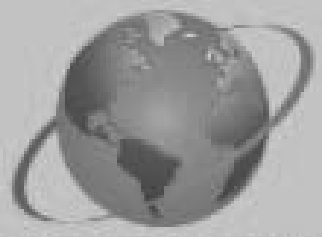

OMNIS SCIENTIA

\section{SAÚDEE PÚBUICA BM}

\section{THEMIPOS PANDENMICOS}

\section{VOLUME 1}

Organizador:

Raul Sousa Andreza

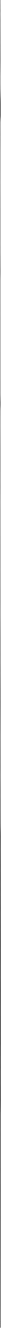


Editora Omnis Scientia

SAÚDE PÚBLICA EM TEMPOS PANDÊMICOS

Volume 1

$1^{a}$ Edição

TRIUNFO - PE

2021 


\section{Editor-Chefe}

Me. Daniel Luís Viana Cruz

Organizador (a)

Prof. Me. Raul Sousa Andreza

\section{Conselho Editorial}

Dra. Pauliana Valéria Machado Galvão

Dr. Wendel José Teles Pontes

Dr. Walter Santos Evangelista Júnior

Dr. Cássio Brancaleone

Dr. Plínio Pereira Gomes Júnior

Editores de Área - Ciências da Saúde

Dra. Camyla Rocha de Carvalho Guedine

Dra. Cristieli Sérgio de Menezes Oliveira

Dr. Leandro dos Santos

Dr. Hugo Barbosa do Nascimento

Dr. Marcio Luiz Lima Taga

Dra. Pauliana Valéria Machado Galvão

\section{Assistentes Editoriais}

Thialla Larangeira Amorim

Andrea Telino Gomes

Imagem de Capa

Freepik

\section{Edição de Arte}

Vileide Vitória Laranjeira Amorim

\section{Revisão}

Os autores

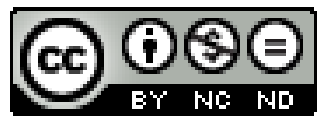

Este trabalho está licenciado com uma Licença Creative Commons - AtribuiçãoNãoComercial-SemDerivações 4.0 Internacional.

O conteúdo abordado nos artigos, seus dados em sua forma, correção e confiabilidade são de responsabilidade exclusiva dos autores. 
Dados Internacionais de Catalogação na Publicação (CIP) (eDOC BRASIL, Belo Horizonte/MG)

S255 Saúde pública em tempos pandêmicos [livro eletrônico] / Organizador Raul Sousa Andreza. - Triunfo, PE: Omnis Scientia, 2021.

286 p. : il.

Formato: PDF

Requisitos de sistema: Adobe Acrobat Reader

Modo de acesso: World Wide Web

Inclui bibliografia

ISBN 978-65-88958-41-4

DOI 10.47094/978-65-88958-41-4

1. Ciências da saúde. 2. Saúde pública. 3. Pandemia. I. Andreza, Raul Sousa.

CDD 610

Elaborado por Maurício Amormino Júnior - CRB6/2422

\author{
Editora Omnis Scientia \\ Triunfo - Pernambuco - Brasil \\ Telefone: +55 (87) 99656-3565 \\ editoraomnisscientia.com.br \\ contato@editoraomnisscientia.com.br
}




\section{PREFÁCIO}

A incidência do novo coronavírus no Brasil é preocupante. No entanto, a saúde pública do país e o sistema de atendimento visa abranger a diversidade que o Brasil apresenta, o sistema único de saúde (SUS) tem como base a integralidade, a universalidade e a equidade de todos os pacientes e trabalhadores. Instituído assim, para democratizar toda a saúde brasileira, tem o interesse de ofertar serviços de qualidade a população. Portanto, ao longo da história de sua consolidação a saúde pública foi deixado de lado e passou a gerar grandes problemas aos profissionais atuantes.

De fato, os estudos desenvolvidos no âmbito da saúde pública se propõem a articular conhecimentos de diferentes saberes e fazeres fornecendo subsídios teóricos, práticos e metodológicos que contribuem positivamente para a construção de estratégias e políticas públicas que visam o desenvolvimento de informações e ações em prol de uma saúde de qualidade para toda comunidade.

O presente livro é composto por 24 capítulos elaborados por autores pertencentes às ciências da saúde e suas áreas afins com o objetivo de proporcionar conhecimentos e compartilhar experiências e resultados de estudos desenvolvidos em várias localidades brasileiras e que visam à elucidação de diferentes situações de saúde.

Em nossos livros selecionamos um dos capítulos para premiação como forma de incentivo para os autores, e entre os excelentes trabalhos selecionados para compor este livro, o premiado foi o capítulo 02, intitulado "VACINAS CONTRA COVID-19: UMA BREVE DESCRIÇÃO POR MEIO DE REVISÃO INTEGRATIVA“. 


\section{SUMÁRIO}

CAPÍTULO 1

A IMPORTÂNCIA E OS DESAFIOS DA ESTRATÉGIA DE SAÚdE DA FAMÍLIA NO ENFRENTAMENTO DA COVID-19

Romana Erica Tavares Grangeiro Pinto

Wyara Ferreira Melo

Maria Amanda Laurentino Freires

Patrício Borges Maracajá

Aline Carla de Medeiros

José Cândido da Silva Nóbrega

Manoel Marques de Souto Nóbrega Filho

Túlio Alberto de Oliveira Sousa

Mônica Valéria Barros Pereira

Vicente Saraiva dos Santos Neto

Hozanna Estrela Celeste

Gabriela Rocha Pordeus dos Santos

DOI: 10.47094/978-65-88958-41-4/17-26

CAPÍTULO 2

VACINAS CONTRA COVID-19: UMA BREVE DESCRIÇÃO POR MEIO DE REVISÃO INTEGRATIVA

Fernanda Lima Marçal

Isabela Figueiredo e Souza

Maria Eduarda Coelho Gomes

Larissa Lima Torres

Isabela Campbell Santos

Thamara Lóren Lima

Ludmilla Vieira Magalhães

Maria Eduarda Sirina Pereira

Lucas Viana de Oliveira 
Larissa da Silva Torres França

Natan Fiorotti da Silva

Milena de Oliveira Simões

DOI: 10.47094/978-65-88958-41-4/27-41

CAPÍTULO 3

AÇÃO EM SAÚdE E A IMPORTÂNCIA DO USO CORRETO DAS MÁSCARAS PARA PREVENÇÃO DE INFECÇÕES RESPIRATÓRIAS

Soniely Nunes de Melo

Rafael Belarmino de Souza Lima

Tarcísio Correia Sposito

Rayana Ribeiro Trajano de Assis

Nayara Sandriele Santana de Souza

Bruna Rafaella Santos Torres

Flávio José Alencar de Melo

Davi Silva de Jesus

Carlos Henrique Bezerra de Siqueira

Izabelle Barbosa da Silva

Marcos André de Holanda Prudente Pessoa

Ana Marlusia Alves Bomfim

DOI: 10.47094/978-65-88958-41-4/42-51

CAPÍTULO 4

CONHECIMENTOS DAS EQUIPES MULTIPROFISSIONAIS DA ESTRATÉGIA DE SAÚDE DA FAMIILIA SOBRE COVID-19 E GESTAÇÃO

Priscilla dos Santos Nascimento

Michelle Araújo Moreira

DOI: 10.47094/978-65-88958-41-4/52-65 
CARTILHA EDUCATIVA SOBRE A COVID-19 PARA A PROTEÇÃO DA COMUNIDADE ACADÊMICA DO CAMPUS BELÉM DO IFPA

Lidineusa Machado Araujo

Maria de Nazaré Pereira Rodrigue Martins

Gabriela Priscila de Lima Carvalho

Fernanda Rafaela de Souza Rebelo da Costa

Michelle da Silva Pereira

Andréa de Melo Valente

Maria Helena Cunha Oliveira

Antônio Marcos Mota Miranda

DOI: 10.47094/978-65-88958-41-4/66-75

CAPÍTULO 6 .76

A SAÚdE DOS PROFISSIONAIS DE SAÚDE QUE ATUAM NA LINHA DE FRENTE NA PANDEMIA DO COVID - 19: REVISÃO INTEGRATIVA

João Lucas Ferreira Andrade

Léa Bianch Lima

Luana Kellen Nogueira Epitácio

Maria Eduarda Alves Vasconcelos

Antônio Augusto Ferreira Carioca

Carlos Antônio Bruno da Silva

Eudóxia Sousa de Alencar

DOI: 10.47094/978-65-88958-41-4/76-89

CAPÍTULO 7 .90

OS EFEITOS DA PANDEMIA NA COMPULSÃO ALIMENTAR: UMA REVISÃO DE LITERATURA

Lais Pontes de Miranda Cerqueira

Tarcio Goncalves sobral

Isadora Bianco Cardoso de Menezes

DOI: 10.47094/978-65-88958-41-4/90-97 
O IMPACTO DA PANDEMIA DO COVID - 19 SOBRE A SEGURANÇA ALIMENTAR E NUTRICIONAL: REVISÃO INTEGRATIVA

Bruna Sousa Barbosa

Igor Matheus Cruz de Oliveira

João Lucas Ferreira Andrade

Léa Bianch Lima

Luana Kellen Nogueira Epitácio

Maria Eduarda Alves Vasconcelos

Antônio Augusto Ferreira Carioca

Carlos Antônio Bruno da Silva

Eudóxia Sousa de Alencar

DOI: 10.47094/978-65-88958-41-4/98-104

CAPÍTULO 9 105

A UTILIZAÇÃO DOS PROTOCOLOS DE USO DO GUIA ALIMENTAR PARA A POPULAÇÃO BRASILEIRA: RELATO DE EXPERIÊNCIA

Bárbara Santos Amorim

Lis Chaves Marinho

Isadora Bianco Cardoso de Menezes

DOI: 10.47094/978-65-88958-41-4/105-114

CAPÍTULO 10 .

O IMPACTO DA COVID-19 EM PORTADORES DE SÍNDROME METABÓLICA

Laura Rasul de Lima

Ana Beatriz Amaral Vieira

Gabriella Neiva Reis

Ingrid Ravenna Liberalino Lima

DOI: 10.47094/978-65-88958-41-4/115-122 
SIMPLIFICANDO A HANSENÍASE: PROJETO DE INTERVENÇÃO REALIZADO EM

MEIO DIGITAL DURANTE A PANDEMIA

Amanda Almeida Lima

Ana Beatriz Sousa Santos

Francisco Vittor Miranda e Araújo

Jesamar Correia Matos Filho

João Ferreira de Paula Neto

Maria Clara de Freita Albano

Manoel Cícero Viana de Lima

Pedro Schmitt Martins Paiva Matos

Ruddy Mariano Maia Cysne Guerra

Samuel Carvalho Vasconcelos

Thaine Mirla Rocha

Elaine Lopes Bomfim

DOI: 10.47094/978-65-88958-41-4/123-131

CAPÍTULO 12.

DESCOMPLICANDO A HANSENÍASE: PROJETO DE INTERVENÇÃO REALIZADO EM UNIDADE DE SAÚDE NO PERÍODO DA PANDEMIA

Adália Stefanny de Araujo Cavalcante

Giovanna Giffoni Souza do Nascimento

Iêda de Freitas Martins Jota

Isabel Camila Araújo Barroso

Kaio Rangel Freitas Guimarães

Laís Mesquita de Sousa

Monique dos Santos Chaves

Manoel Victor Freires Vieira

Matheus Macedo Braga Coelho

Thaine Mirla Rocha

Elaine Lopes Bomfim 
CAPÍTULO 13. 140

HANSENÍASE: UM ESTUDO EPIDEMIOLÓGICO NA REGIÃO DO MÉDIO ARAGUAIAMATO GROSSO

Flavia Rodrigues Santana

Josilene Dália Alves

DOI: 10.47094/978-65-88958-41-4/140-150

CAPÍTULO 14 151

MUNICÍPIOS COM ALTA INCIDÊNCIA DE TUBERCULOSE EM MATO GROSSO: CONHECER PARA INTERVIR EM TEMPOS DE PANDEMIA

Larissa Machado Bellé

Yasmim Paloma Abreu Silva

Alessandro Rolim Scholze

Josilene Dália Alves

DOI: 10.47094/978-65-88958-41-4/151-162

CAPÍTULO 15 163

SAÚDE INTEGRAL DA MULHER EM CONTEXTO PANDÊMICO: REVISÃO INTEGRATIVA DE LITERATURA

Lohana Guimarães Souza

Tailande Venceslau Carneiro

Letícia Grazielle Santos

DOI: 10.47094/978-65-88958-41-4/163-175

CAPÍTULO 16 176

ASSISTÊNCIA DE ENFERMAGEM NO OUTUBRO ROSA E PERFIL EPIDEMIOLÓGICO DAS MULHERES: RELATO DE EXPERIÊNCIA

Helena Pereira de Souza

Laura Letícia Perdição Guerra

Luana Fernandes e Silva 
Thales Philipe Rodrigues da Silva

Alessandra Lage Faria

Helen Carine Ferreira Balena

Érica Moreira de Souza

Bruna Luíza Soares Pinheiro

Lorena Medeiros de Almeida Mateus

Flávia Duarte de Oliveira Ribeito

Bianca Maria Oliveira Luvisaro

Fernanda Penido Matozinhos

DOI: 10.47094/978-65-88958-41-4/176-188

CAPÍTULO 17 189

AGRAVAMENTO DAS DOENÇAS PSIQUIÁTRICAS DURANTE O PERÍODO DE ISOLAMENTO SOCIAL: UMA BREVE REVISÃO DE LITERATURA

Hellen Kristina Magalhães Brito

Gabriela Teixeira Lima

Ana Laura Fernandes Tosta

Laura Beatriz Caitano de Oliveira

Maria Paula Ricardo Silva

Mariana Vieira Garcia de Carvalho

Nathália Siriano Costa

Mayara Rita Figueredo

Mabel Fernandes Rocha

Helena Maria Mendes Marques

Kaio Murilo Santana Corrêa

Ana Flávia Buiatte Andrade

DOI: 10.47094/978-65-88958-41-4/189-200 
ATUAÇÃO DO PSICÓLOGO EM TEMPO DE PANDEMIA: UMA REVISÃO DE ESTUDOS NACIONAIS

Gabriel Rigamonte

Sueli Souza

Wilson Quiroz

Daniel Bartholomeu

Fernando Pessotto

Cintia Heloína Bueno

Fernanda Helena Viana Garcia

DOI: 10.47094/978-65-88958-41-4/201-213

CAPÍTULO 19

CRIAÇÃO DE UM APLICATIVO VOLTADO PARA UMA UNIDADE BÁSICA DE SAÚdE EM FORTALEZA

Isabella Araujo Duarte

Giovanna Rolim Pinheiro Lima

Idna Lara Goes de Sena

Laura Figueiredo Leite

Letícia Cavalcante Lócio

Livian Araújo Camelo Gomes

Maria Regina Cardoso Linhares Oliveira Lima

Maria Tereza Linhares Cardoso

Pedro Henrique Cardoso Nogueira

Rafael Albuquerque Franco

Rodrigo Carvalho Paiva

Berta Augusta Faraday Sousa Pinheiro

DOI: 10.47094/978-65-88958-41-4/214-229 
TELEMEDICINA E SUAS VARIÁVEIS NO CENÁRIO DE PANDEMIA MUNDIAL: UMA REVISÃO DE LITERATURA

Raniere Canteiro Garcia Lhamas

Andressa Marcolino Campos

Douglas Ferreira Lima

Gabriel Souza Ferreira Oliveira

Guilherme de Mendonça Lopes Beltrão

Luciana de Paula Santana

Nícollas Nunes Rabelo

DOI: 10.47094/978-65-88958-41-4/230-237

CAPÍTULO 21 238

MONITORIA ACADÊMICA NO ENSINO REMOTO: PERSPECTIVAS E DESAFIOS EM TEMPOS PANDÊMICOS

Felipe Gabriel Assunção Cruz

Givanildo Bezerra de Oliveira

Marcílio Delan Baliza Fernandes

Ana Lúcia Moreno Amor

DOI: 10.47094/978-65-88958-41-4/238-248

CAPÍTULO 22

A DOENÇA DO OLHO SECO NA SÍINDROME DE SJÖGREN

Bruna Rafaella Santos Torres

Carlos Eduardo Ximenes da Cunha

Carlos Henrique Bezerra de Siqueira

Flavia Emanuelly Alves França Gomes

Santília Tavares Ribeiro de Castro e Silva

Anna Caroline Guimarães Gomes

Laís Rytholz Castro

Dennis Cavalcanti Ribeiro Filho 
Lara Medeiros Pirauá de Brito

Marina Viegas Rezende Ribeiro

DOI: 10.47094/978-65-88958-41-4/249-259

CAPÍTULO 23 260

FEIRAAGROECOLÓGICA: DIFICULDADES E POTENCIALIDADES DE UM CIRCUITO CURTO DE COMERCIALIZAÇÃO

Maria Rita Garcia de Medeiros

Rônisson Thomas de Oliveira Silva

Maria Natalícia de Lima

Ana Beatriz Macêdo Venâncio dos Santos

DOI: 10.47094/978-65-88958-41-4/260-269

CAPÍTULO 24 270

AVALIAÇÃO DO IMPACTO DE UMA REDE SOCIAL EM USUÁRIOS DE UMA UNIDADE BÁSICA DE SAÚDE EM FORTALEZA

Luiz Gerson Gonçalves Neto

Letícia Cavalcante Lócio

Carlos Alexandre Leite Pereira Filho

Henrique Sousa Costa

Maria Helena dos Santos Macedo

Lígia Bringel Olinda Alencar

Berta Augusta Faraday Sousa Pinheiro

Isaac Dantas Sales Pimentel

DOI: 10.47094/978-65-88958-41-4/270-280 
TELEMEDICINA E SUAS VARIÁVEIS NO CENÁRIO DE PANDEMIA MUNDIAL: UMA REVISÃO DE LITERATURA

Raniere Canteiro Garcia Lhamas';

Centro Universitário Atenas (UniAtenas) - Paracatu, MG.

http://lattes.cnpq.br/7036193851759792

Andressa Marcolino Campos²;

Centro Universitário Atenas (UniAtenas) - Paracatu, MG.

http://lattes.cnpq.br/4527851523834449

\section{Douglas Ferreira Lima³;}

Centro Universitário Atenas (UniAtenas) - Paracatu, MG.

http://lattes.cnpq.br/3907403493460590

\section{Gabriel Souza Ferreira Oliveira ${ }^{4}$;}

Centro Universitário Atenas (UniAtenas) - Paracatu, MG.

http://lattes.cnpq.br/8957862638287193

\section{Guilherme de Mendonça Lopes Beltrão;}

Faculdade Ciências Médicas de Minas Gerais (CMMG) - Belo Horizonte, MG.

http://lattes.cnpq.br/6275541936327273

\section{Luciana de Paula Santana ${ }^{6}$;}

http://lattes.cnpq.br/1878274846555615

Faculdade Ciências Médicas de Minas Gerais (CMMG) - Belo Horizonte, MG.

Nícollas Nunes Rabelo?.

Departamento de Neurocirurgia. Centro Universitário Atenas - Paracatu, MG.

RESUMO: A telemedicina, no sentido geral, viabiliza a oferta de serviços relacionados à saúde através do uso de tecnologias de informação e comunicação, tendo grande relevância em casos nos quais a distância é um fator crítico, como na atual situação pandêmica. Nessa conjuntura, a telemedicina vem conquistando grande espaço no cenário contemporâneo do Brasil, apesar de já ser utilizada em países desenvolvidos, por meio de dispositivos de medição biométrica, como medidores de glicose, frequência cardíaca e pressão arterial. A telemedicina ou telessaúde, mais do que um recurso tecnológico para proporcionar a realização de atividades a distância, adquire efetividade quando está associada a planos estratégicos que incluam um processo de logística de distribuição de serviços 
de saúde. Sua vinculação com estratégias é devido à necessidade de a telemedicina estar inserida dentro de um plano global de ação, considerando-se fatores como tempo (momento) e espaço (local geográfico). Isso significa que a telemedicina deve estar contextualizada em relação ao momento temporal e às características da localidade onde será implantada, para que seja possível definir os tipos de atividade a serem realizadas. Inserir a telemedicina numa estratégia significa colocá-la numa posição exclusiva e valiosa.

PALAVRAS-CHAVE: Telemedicina. Tecnologia. Saúde.

\section{TELEMEDICINE AND ITS VARIABLES IN THE WORLD PANDEMIC SCENARIO: A LITERATURE REVIEW}

ABSTRACT: Telemedicine, in the general sense, enables the provision of health-related services through the use of information and communication technologies, having great relevance in cases in which distance is a critical factor, as in the current pandemic situation. In this context, telemedicine has been gaining great space in the contemporary scenario of Brazil, although it is already used in developed countries, using biometric measurement devices, such as glucose, heart rate and blood pressure meters. Telemedicine or telehealth, more than a technological resource to provide remote activities, becomes effective when it is associated with strategic plans that include a logistics process for the distribution of health services. Its link with strategies is due to the need for telemedicine to be part of a global action plan, considering factors such as time (moment) and space (geographic location). This means that telemedicine must be contextualized in relation to the temporal moment and the characteristics of the location where it will be implanted, so that it is possible to define the types of activities to be performed. Inserting telemedicine in a strategy means placing it in an exclusive and valuable position.

KEY-WORDS: Telemedicine. Tecnology. Health.

\section{INTRODUÇÃO}

A telemedicina, caracterizada pelo uso de tecnologias informativas e comunicativas na área da saúde humana, possibilita a oferta de serviços relacionados com aperfeiçoamento da obtenção de cuidados pelo paciente. Com a evolução secular dos processos tecnológicos até o século XXI, houve o advento de capacidades tecnológicas e, interligando-as ao sistema de prestação de saúde, proporcionou oportunidades de aprimorar o atendimento clínico vigente. (ZANOTTO et al., 2020)

O primeiro marco para a consolidação no Brasil foi o lançamento da telemedicina como demanda induzida no edital de 2005 do Programa "Institutos do Milênio". Para tal objetivo, o CNPq (Conselho Nacional de Desenvolvimento Científico e Tecnológico) desenvolveu ambientes de tutoria eletrônica e ambulatórios virtuais, estabeleceu diversas 
parcerias, promoveu treinamento nas instituições e em órgãos governamentais como o Ministério da Saúde e fomentou o surgimento de novos núcleos. (WEN, 2008)

A telemedicina, além de agregar eficiência e reduzir custos, pode ampliar a atenção primária, significando o acesso a serviços de saúde em regiões remotas, uma vez que tem o potencial de ampliar as ações dos profissionais de saúde, integrando-os aos serviços de saúde localizados em hospitais e centros de referência, no que tange à prevenção, diagnóstico e tratamento. Nesse sentido, o aspecto primordial da telemedicina é o seu potencial de democratizar o acesso aos serviços de saúde: do ponto de vista social, representa uma atividade de grande importância, em especial por ser referenciada crescentemente na literatura relacionada ao aumento de qualidade e segurança na prestação dos cuidados médicos e também às reformas necessárias aos sistemas de saúde universais. (WEN, 2008)

Assim, compreende-se que há uma insuficiência médica para atender a alta demanda presencial em regiões geográficas específicas, uma vez que foi observado por Zanotto et al. (2020) a necessidade de consulta oftalmológica especializada de 10 vezes maior que a real disponibilidade no território analisado. Apesar de o país possuir condições relevantes para o desenvolvimento e aplicação da telemedicina, em virtude de seu amplo território terrestre, percebe-se que esse meio tecnológico foi pouco abrangido na área médica, possuindo hesitação acerca de sua capacidade benéfica e de segurança ao paciente. Desse modo, o presente trabalho objetiva discorrer acerca da vantagens no âmbito social, econômico e de saúde para a sociedade.

\section{METODOLOGIA}

Para o desenvolvimento do artigo, realizou-se uma pesquisa minuciosa de artigos científicos embasados acerca do tema telemedicina, principalmente aqueles datados das últimas duas décadas. Para a seleção dos estudos, utilizou-se a plataforma eletrônica virtual do Pubmed, Scielo, Elsevier e Periódicos CAPES. Dos 15 artigos pré-selecionados, nove foram escolhidos para estudo aprofundado e abordagem no trabalho, uma vez que possuíam melhor conteúdo sobre o tema e suas variações, seja em âmbitos financeiros, sociais ou de promoção da saúde nos indivíduos.

\section{RESULTADOS E DISCUSSÃO}

A partir do levantamento bibliográfico realizado nesse trabalho, fez-se uma síntese do material encontrado em 9 artigos científicos, considerando diversos setores do tema em questão.

De acordo com Zanotto et al. (2020), a grande população mundial existente e o constante uso de recursos, sendo necessário medidas de sustentabilidade, promoveu maior interesse na rede ampla de possibilidades da telemedicina. Dentre esses, destaca-se o seu papel no âmbito da atenção primária à saúde (APS) por meio do rápido acesso de pacientes às referências de alta complexidade, da possível resolubilidade no nível de atenção básica e da redução de encaminhamentos para atendimentos especializados, além de sua capacidade de auxiliar no monitoramento e controle do valor de entrega 
do cuidado ao indivíduo.

$\mathrm{Na}$ literatura em geral, é atribuído a telemedicina um enorme potencial de promover e agregar benefícios socioeconômicos à sociedade à medida que: promove o acesso aos serviços de saúde, cria oportunidades de aprimoramento (educação) para os profissionais e melhora a atenção e qualidade de vida, além é claro, ajudar na organização dos provedores (instituições e empresas). (MEDEIROS; JACQUES, 2004)

O Conselho Federal de Medicina (CFM) define a telemedicina, pelo documento redigido em 2002, como sendo a atividade da profissão médica por meio do uso de metodologias de interação comunicativa áudio-visual e de dados, no qual se tem propósito de proporcionar assistência, educação e pesquisa em saúde. No entanto, para sua efetividade no cenário atual, deve-se seguir as normas estabelecidas pelo conselho de guarda, confidencialidade e privacidade de dados, além de assegurar o sigilo do profissional médico e de fundamentar no benefício do indivíduo. (Resolução CFM n ${ }^{\circ}$ $1.643 / 2002 ; 2002$, p. 205.)

Ademais, segundo a Resolução CFM no 1.643/2002 (2002, p. 205.), os prestadores de serviço à distância são obrigados a realizar inscrição no Cadastro de Pessoa Jurídica do Conselho Regional de Medicina da respectiva localidade em que se encontra, visto que esse órgão estabelecerá vigilância contínua a respeito da qualidade da atenção à saúde, bem como da preservação de informações e da relação apropriada entre paciente e profissional.

Segundo Wen (2008), pode-se agrupar as atividades da telemedicina em três grandes conjuntos:

1. Teleducação Interativa e Rede de Aprendizagem Colaborativa: são termos que designam o uso de tecnologias interativas para ampliar as possibilidades de construção de conhecimentos, seja aumentando as facilidades de acesso a materiais educacionais de qualidade, seja permitindo acesso a centros de referência ou a estruturação de novas sistemáticas educacionais.

2. Teleassistência/Regulação e Vigilância Epidemiológica: desenvolvimento de atividades com fins assistenciais à distância, tais como a segunda opinião especializada. Podem ser desenvolvidos sistemas para permitir a integração de atividades assistenciais com educação, vigilância epidemiológica e gestão de processos em saúde.

3. Pesquisa Multicêntrica/Colaboração de Centros de Excelência e da Rede de Teleciência: integração de diversos centros de pesquisa, permitindo a otimização de tempo e de custos, por meio do compartilhamento de dados, da capacitação e da padronização de métodos.

A educação é um processo complexo e, atualmente, com a facilidade de acesso às tecnologias, ela pode ganhar um reforço, potencializando os métodos educacionais clássicos. Existem diversas tecnologias interativas de apoio, seja para a educação presencial, seja para a Teleducação Interativa ou Ensino à Distância (EaD). Entre elas, podemos citar a computação gráfica, os simuladores cirúrgicos, o ambiente de simulação realística e o laboratório de habilidades com manequins robóticos, entre outros. (WEN, 2008). 
Sobre o viés econômico, analisado por Zanotto et al. (2020), os gastos necessários para a introdução desses mecanismos tecnológicos são altos, sendo necessário métodos de microcusteio para valoração de custos com elevado grau de detalhes. Nesse sentido, o Time-Driven Activity-Based Costing (TDABC) é uma alternativa de mensuração que permite relacionar o tempo essencial para efetivar a consulta, além do preço unitário do serviço.

Um estudo realizado no Rio Grande do Sul acerca de mensuração de valores de consultas pela equipe profissional na área oftalmológica, denominado atendimento teleoftalmológico, demonstrou que o custo aferido padrão por telediagnóstico na área era de R \$ 199,00. Foi-se avaliado durante o período de um ano todo o processo e definiu-se que houve um decréscimo do custo de $\mathrm{R} \$ 783,00$ para $\mathrm{R} \$ 283,30$, o que determina a curva de aprendizado de implementação da atual tecnologia. (ZANOTTO et al., 2020)

Além da questão financeira, a telemedicina possui possibilidade de qualificar o sistema de saúde, promovendo aperfeiçoamento do acesso ao serviço por intermédio de uma maior resolubilidade da APS, fato que repercute diretamente no setor secundário e terciário, sendo positivo para a diminuição de encaminhamento para esses. Dessa maneira, em conformidade com Zanotto et al. (2020), os sistemas de teleoftalmologia são viáveis no cenário brasileiro, sendo de fundamental importância a respeito da qualificação da fila de espera de atendimentos nessa área na atenção primária à saúde. Por meio dela, é possível realizar a classificação do risco do paciente, fato que permite o melhor acesso à especialidade e o cuidado ao indivíduo.

Diante da pandemia de COVID-19 presenciada no Brasil e mundialmente, o Conselho Federal de Medicina determinou o aprimoramento da capacidade qualitativa dos serviços médicos realizados pelos profissionais, reconhecendo a utilidade benéfica da telemedicina. Além do prescrito na Resolução CFM n ${ }^{\circ}$ 1.643, de 26 de agosto de 2002, determinou-se: a teleorientação de pacientes à distância, com instrução e encaminhamento de indivíduos em isolamento; telemonitoramento, exercido sob supervisão do profissional da saúde para controlar parâmetros de saúde ou doença; e teleinterconsulta, sendo realizado de forma a permutar informações entre médicos, para assistência diagnóstica ou terapêutica. (Ofício CFM nº 1756/2020 - COJUR; 2020)

No âmbito da genética, a telemedicina possui característica relevante no processo de atendimento de maneira eficiente à população, uma vez que a telegenética possibilita atingir e ultrapassar barreiras geográficas e laborais na região, possibilitando uma maior eficiência dos custos. Dessa maneira, uma vez movimentando a informação ao invés do paciente, a telemedicina propicia um ambiente favorável ao avanço de cuidados de saúde e retira impasses acerca do não fornecimento de serviços na área genética, em especial no aconselhamento genético em oncologia e na síndrome da predisposição genética a câncer. (FERNANDES TAVARES et al., 2020).

Em um estudo realizado a respeito do assunto nos Estados Unidos da América (EUA), Austrália e País de Gales, foi observado, por Fernandes Tavares et al. (2020), baixas desvantagens do método virtual, elevando a possibilidade de sua instalação de forma contínua. Dentre as vantagens obtidas pelos pacientes do trabalho, destacam-se: baixo custo, ausência de locomoção prolongada até grandes centros, possibilidade de agendamento do aconselhamento genético em conjunto com uma 
consulta presencial, facilidade de transmissão de informações e acesso a áreas rurais antes ausentes de serviços. Ainda, as desvantagens do processo são: falta de apoio emocional e de vínculo afetivo entre médico e paciente.

A telemedicina deve ser aplicada com médicos especialistas, ou seja, quando possui Registro de Qualificação de Especialista (RQE), e pode ser aplicado quando o paciente está em áreas vulneráveis ou de difícil acesso ao centro de saúde, em situações, como desastres naturais, prisões, estações especiais e asilos. (MALDONADO; MARQUES; CRUZ, 2016)

Segundo Maldonado, Marques e Cruz (2016), duas especialidades se destacam, sendo a teleradiologia e teledermatogia. A teleradiologia depende da opinião do radiologista que analisa imagens de Raios-X e de tomografia computadorizada, os exames são feitos em áreas de poucos recursos e posteriormente encaminhados para centros de saúde mais avançados. Houve uma comprovação de que as análises por meio digital foram mais eficazes quando associada às imagens originais. Já na área de dermatologia o uso de imagens transferidas para o médico houve acertos nos diagnósticos, e a principal doença identificada foi de Hanseníase, e 95,8\% dos diagnósticos foram efetivos.

Na Faculdade de Medicina da Universidade de São Paulo, o seu grupo de Telemedicina, criou um web site sobre a prevenção do câncer de pele e Tele-Educação em Microbiologia. O web site teve como objetivo a preparação de indivíduos não especialista na área, na detecção de possíveis lesões malignas e influenciar a busca de um profissional. Além do mais, busca a promover um intercâmbio multiprofissional em microbiologia, para fiscalizar o surgimento de resistência bacteriana. (URTIGA; LOUZADA; COSTA, 2004)

Em outubro de 2000, o Instituto Marteno Infantil de Pernambuco (IMIP), foi estabelecido um programa internacional de telepatologia entre o IMIP e o St. Jude Chidren's Research Hospital nos EUA. O projeto teve como propósito um estudo simultâneo entre os patologistas das duas instituições acerca do diagnóstico de câncer pediátrico, o que teve comunicação em tempo real e na destinação de imagens estáticas. Segundo Urtiga, Louzada e Costa (2004), o resultado inicial mostrou que foi um procedimento viável, o que possibilitou na rapidez e no aumento de qualidade no tratamento de crianças com câncer.

Além disso, a telemedicina contribui para que os médicos possam interpretar exames a longa distância por causa do uso da câmera, no caso a transmissão de dados digitais, por exemplo, ECG, EEG, ultrassonografia e monitoramento de sinais vitais. Isso facilita a rapidez do diagnóstico e conduta que o médico deve ter com o paciente, mas também evita deslocamentos excessivos com esses indivíduos. (CL, 2015)

Ademais, esse modelo de aconselhamento genético por meio virtual estudado demonstra que tal método pode ser utilizado tanto em países subdesenvolvidos e em desenvolvimento, nos quais existem maiores dificuldades de acesso dos serviços de saúde em sua totalidade. Portanto, é impreterível a análise do caso do indivíduo em questão pelo profissional de saúde, sendo que a telemedicina não deve substituir o trabalho do profissional médico e, sim, adequar-se como instrumento de assistência. (FERNANDES TAVARES et al., 2020). 


\section{CONCLUSÃO}

Diante do cenário observado, a telemedicina pode se tornar um grande complemento no processo de saúde. Dentre os pontos positivos observados estão o seu grande alcance geográfico, diminuindo as barreiras da distância entre o paciente e o médico escolhido/adequado para o seu tratamento, além do destaque quanto ao menor custo em relação às consultas ou procedimentos realizados de forma presenciais. Além disso, um adentro que chama a atenção é a facilitação da relação médico-paciente, já que o contato entre os mesmos pode se tornar mais frequente é viável mediante à menor dificuldade encontrada em questões burocráticas como deslocamento, salas de espera e outras situações destacadas por usuários deste novo tipo de serviço. Considerando esse panorama, a telemedicina surge como uma alternativa de oferecimento de uma gama de serviços os quais por muitas vezes, estavam restritos apenas à grandes centros.

Com isso, é visível também um grande trunfo do Sistema Único de Saúde (SUS) na busca da saúde integral, haja visto que o atendimento a distância pode acrescentar de forma direta na atenção primaria e na utilização de diversos serviços para atender remotamente pessoas que vivem em locais com pouca estrutura física ou com uma quantidade restrita de profissionais especialistas, ou ainda regiões em que o deslocamento do paciente é inacessível na busca de solução para o seu problema.

\section{REFERÊNCIAS}

WEN, Chao Lung. Telemedicina e telessaúde - um panorama no Brasil. Informática Pública ano 10, 2008.

ZANOTTO, B. S. et al. Avaliação Econômica de um Serviço de Telemedicina para ampliação da Atenção Primária à Saúde no Rio Grande do Sul: o microcusteio do Projeto TeleOftalmo. Ciência \& Saúde Coletiva, v. 25, n. 4, p. 1349-1360, 1 abr. 2020.

MEDEIROS, Rogério; JACQUES, Wainer. Telemedicina: onde estão seus benefícios sócioeconômicos?. Unifesp, 2004.

Conselho Federal de Medicina (BR). Resolução CFM nº 1.643/2002 [Internet]. Diário Oficial da União, Brasília, DF; 2002 ago. 26. Seção I, p. 205.

MALDONADO, Jose Manuel Santos de Varge; MARQUES, Alexandre Barbosa; CRUZ, Antonio. Telemedicina: desafios à sua difusão no Brasil. Cad. Saúde Pública, Rio de Janeiro, v. 32, supl. 2, e00155615, 2016.

Conselho Federal de Medicina (BR). Ofício CFM n 1756/2020 - COJUR [Internet]. Brasília: Conselho Federal de Medicina; 2020.

FERNANDES TAVARES, D. et al. A Utilização de Telemedicina para o Aconselhamento Genético em Oncologia. Revista Brasileira de Cancerologia, v. 66, n. 1, 16 mar. 2020.

URTIGA, Keylla Sá; LOUZADA, Luiz AC; COSTA, Carmen Lúcia B. Telemedicina: uma visão geral do estado da arte. São Paulo-SP: Universidade Federal de São Paulo/Escola Paulista de Medicina 
(UNIFESP/EPM), 2004.

CL, Wen. Telemedicina e Telessaúde: oportunidade de novos serviços e da melhoria da logística em saúde. Rev. Panorama Hospitalar [Internet], 2015. 


\section{Índice Remissivo}

$\mathbf{A}$

Acesso aos psicólogos 201, 203, 204

Acesso às redes sociais 43

Agricultor e o consumidor 260

Agricultores familiares 260, 263, 266

Alterações do metabolismo 115

Ambiente virtual 215

Ansiedade 77, 78, 81, 82, 83, 84, 86, 87, 91, 92, 94, 95, 117, 121, 190, 192, 193, 194, 197, 204, 207, 208, 209

Aplicativo 136, 215, 217, 218, 219, 220, 221, 222, 223, 224, 225, 226, 227, 228, 243, 271, 272, 273, 274, 278, 280

Aprendizagem 239

Assistência social 163, 171, 205

Associação da covid-19 com a gestação 52

Astrazeneca (universidade de oxford) 28, 30

Atenção primária 25, 124

Atenção primária à saúde (aps) 19, 59, 107, 125, 177, 178

Atendimento ambulatorial 105

Atendimento on-line 201, 203

Atendimento remoto 202, 205

Atividades econômicas 28

Autoanticorpos 250

Autoridades sanitárias 67, 69, 73, 278

C

Capacidade de defesa do organismo 115

Características dos imunizantes 28, 30

Cartilha educativa sobre a covid-19 67, 69, 70

Ceratoconjuntivite sicca $(\mathrm{kcs}) 250$

Combate à hanseníase 133

Comercialização 38, 260, 261, 262, 263, 264, 265, 266, 267, 268, 269

Compulsão alimentar 90, 92, 93, 94, 95, 96

Consumo de alimentos 105

Contaminação 43, 44, 45, 47, 49, 72, 73, 74, 78, 117, 135, 179, 191, 193, 195, 197

Coronavac (sinovac) 28, 29

Cuidados individuais e coletivos $67,69,73$

Cuidados preventivos ao covid-19 115

D

Depressão 78, 81, 82, 83, 87, 91, 92, 94, 95, 110, 172, 190, 192, 193, 194, 197, 207, 208, 209

Desemprego 21, 99, 103, 153

Desenvolvimento do câncer 177, 185

Desigualdade em saúde 163

Desinformação 29, 208 
Diabetes 21, 56, 57, 95, 105, 106, 107, 108, 114, 116, 117, 118, 119, 120, 181, 183, 186

Direito humano à alimentação adequada 103, 260

Disseminação da informação 67,73

Distanciamento social 25, 48, 55, 61, 68, 73, 90, 95, 126, 130, 192, 195, 202, 279

Distúrbios psicológicos 190

Doença infecciosa 53, 125, 133, 134, 140, 141, 151, 152

Doença multifatorial 250

Doenças 21, 24, 28, 29, 43, 54, 56, 57, 59, 69, 91, 95, 106, 107, 108, 115, 116, 117, 119, 120, 129, 133, 137, 140, 142, $149,161,163,164,167,171,172,173,177,185,186,193,194,195,207,210,217,222,223,227,250,271$, $272,273,278,279$

Doenças crônicas 105, 171, 218, 221, 227, 273

Doenças negligenciadas 140, 142

\section{$\mathbf{E}$}

Educação em saúde 19, 24, 43, 46, 48, 50, 67, 69, 73, 105, 108, 133, 134, 135, 136, 137, 145, 179, 185, 215, 216, 217, $227,228,238,240,248,271,272,274$

Efeitos colaterais $28,30,31,32,33$

Enfermagem 50, 56, 57, 58, 60, 61, 88, 89, 122, 149, 161, 176, 177, 178, 180, 188, 227, 240, 280

Enfermidade epidêmica 238

Enfrentamento da covid-19 18, 19, 20, 23, 24, 25

Epidemiologia 114, 140, 148, 149, 160, 161, 242, 248

Equipamentos de proteção 24, 43, 47, 78, 171, 197

Equipes multiprofissionais $52,54,55,56,59,60,61,62$

Estratégia saúde da família (esf) 18, 19, 23, 54

Estratégias nutricionais 105

Estresse 77, 78, 81, 82, 83, 86, 87, 88, 89, 94, 172, 190, 192, 193, 197, 206, 207, 209, 210, 211

Etiologia autoimune 250

Evidências científicas 29, 52, 56, 57, 59, 61, 62, 63, 76, 79, 99, 101, 190

F

Feiras agroecológicas 260, 261, 264

Ferramenta tecnológica de saúde 215

Fortalecimento da saúde púbica 67

Frequência cardíaca 230

G

Gestação 52, 53, 54, 57, 58, 59, 61, 62, 63, 224

Glândulas exócrinas 250, 251, 252, 253

Guia alimentar 105, 107, 108, 112, 113

$\mathbf{H}$

Hábitos alimentares 90, 92, 94, 95, 111, 112

Hanseníase 124, 125, 126, 127, 128, 130, 131, 133, 134, 135, 136, 137, 138, 139, 140, 141, 142, 143, 144, 145, 146, $147,148,149$

Herança genética 115

Hipertensão 57, 95, 105, 107, 108, 114, 115, 116, 118, 119, 120, 121, 181, 183, 186, 228, 229, 274, 276,278 
Hiposecreção lacrimal 250

I

Idosos 19, 21, 23, 54, 105, 108, 134, 137

Impacto da pandemia 99

Imunização 28, 29, 30, 35, 38, 179, 183, 186

Inclusão 20, 22, 52, 55, 56, 58, 75, 77, 79, 101, 163, 165, 167, 215, 226, 268

Infecções 28, 29, 38, 43, 44, 48, 57, 59, 60, 78, 165, 172, 180, 227, 251

Infecções respiratórias virais $43,44,48$

Informação de qualidade 271, 279

Informação em saúde 67,69

Informação sobre a hanseníase 124

Instabilidade econômica 99, 103

Instrumento de prevenção 43

Intervenção psicológica 202

Isolamento $23,48,55,60,62,81,90,91,92,94,100,142,164,177,179,190,191,192,193,194,195,196,197,198$, $199,203,204,205,207,208,210,242,261$

Isolamento social 23, 26, 62, 90, 92, 94, 100, 142, 164, 177, 179, 190, 191, 192, 194, 195, 196, 197, 198, 203, 205, 207, 208, 210, 242

$\mathbf{J}$

Janssen (johnson \& johnson) 28, 29, 30

$\mathbf{L}$

Lesões cutâneas 140, 143

Linha de frente 76, 78, 79, 81, 84, 86, 87, 88, 171, 195, 197, 202, 206, 207, 208, 210

M

Má qualidade do sono 77,87

Máscaras faciais 43

Material digital 43, 46

Medição biométrica 230

Medidas de isolamento 26, 28, 194

Medidores de glicose 230

Metodologia pico (problema, intervenção, contexto, resultado) 76, 79, 99, 101

Monitores do centro de ciências da saúde (ccs) 238

Monitoria 238, 239, 240, 241, 242, 243, 244, 246

Morbidade 115, 119

Mortalidade materna 163, 171, 172

Mudanças nos estilos de vida 90, 92, 93

Mycobacterium leprae 135, 140, 141, 146

N

Níveis socioeconômicos 43, 46

Novas modalidades de interações 238

Novo coronavírus sars-cov-2 18, 19 
Olho seco 250, 251, 252, 253, 254, 255, 256, 257, 258

Organização mundial de saúde (oms) 19, 52, 53, 54, 68, 92, 191

Organização pan-americana de saúde (opas) 52, 54

Órgãos oficiais de saúde 238, 247

Outubro rosa $177,178,179,184,185$

$\mathbf{P}$

Padrões dietéticos 115

Pandemia 18, 19, 20, 21, 23, 24, 25, 26, 28, 31, 38, 45, 47, 48, 53, 55, 68, 69, 73, 75, 76, 78, 79, 81, 82, 84, 86, 87, 88, $89,90,91,92,93,94,95,96,99,100,101,102,103,104,117,118,119,120,124,126,130,131,133,135,136$, $137,142,152,153,160,161,162,163,164,165,168,169,170,171,172,173,174,177,179,180,186,187$, 190, 191, 192, 193, 194, 195, 196, 197, 198, 199, 200, 203, 204, 205, 206, 207, 208, 210, 211, 212, 213, 238, $239,240,241,242,244,247,248,271,272,279,280$

Perfil alimentar 105

Perfil clínico 160, 177, 183, 185

Pfizer (pfizer e biontech) 28, 30

Políticas públicas de saúde 18, 24, 173, 204

Portadores de síndrome metabólica 115

Prática oftalmológica 250

Práticas do autocuidado 178,186

Pressão arterial 115,230

Prevenção 29, 33, 72, 170, 187, 221

Primeiros socorros 215, 218, 219, 225, 227, 273

Princípio ativo 28, 30, 33

Produção de alimentos 260

Profissionais de psicologia 202

Profissionais de saúde 18, 22, 24, 54, 56, 76, 78, 79, 81, 82, 84, 85, 86, 87, 89, 107, 125, 138, 151, 160, 170, 185, 194, $195,197,198,202,206,207,208,238$

Programa nacional de alimentação escolar (pnae) 99, 265

Promoção em saúde 43, 45, 271, 278

Propagação do vírus $43,47,48,67,69$

Q

Qualidade de vida 22, 47, 82, 106, 112, 155, 217, 226, 244, 250, 251, 254, 272, 278

Quarentena 90, 100, 121, 126, 170, 172, 190, 191, 192, 193, 194, 195, 199, 202, 206

$\mathbf{R}$

Recurso tecnológico 230

Redes sociais 43, 45, 46, 49, 133, 137, 194, 271, 272, 274, 278, 279

Responsabilidade individual e coletiva 18, 24

Restrição social 90, 92, 93 
Sars-cov- $20,21,29,30,31,32,33,34,35,38,39,40,45,49,53,56,57,58,59,60,65,88,120,163,164,165,168$, $173,191,199,238,239,240,242,243,247,248,279$

Saúde da família 18, 19, 20, 22, 23, 24, 25, 26, 52, 54, 55, 62, 64, 125, 135, 179, 187

Saúde da mulher 163, 166, 173

Saúde de maneira remota 133

Saúde dos profissionais $76,79,81,86,89$

Saúde dos trabalhadores $77,86,87$

Saúde integral 52, 163, 165, 167, 171

Saúde mental 78, 83, 88, 90, 93, 94, 95, 96, 172, 179, 190, 191, 192, 193, 194, 195, 196, 197, 198, 202, 203, 204, 206, $207,208,210,212,222,227,279$

Saúde psicológica 77,87

Saúde pública $6,20,28,38,69,73,82,120,125,134,139,141,148,149,160,168,169,170,171,172,177,179,197$, $199,208,211,238,240,242,247$

Secura ocular 250, 251, 257

Sedentarismo 94, 107, 115, 179

Segurança alimentar e nutricional 99, 100, 101, 102, 103, 104

Segurança alimentar nutricional (san) 99

Serviço de saúde 71, 115, 120, 180

Síndrome de sjögren (ss) 250, 251

Síndrome metabólica 115, 116, 117, 118, 119, 120

Sistema imunológico 58, 115, 117, 120

Sistema nacional de agravos de notificação (sinan) 140, 143

Situação de vulnerabilidade 90, 94, 95, 103

Superfície ocular 250, 254, 258

T

Tecnologia 66, 67, 69, 70, 137, 211, 231, 244

Tecnologias de informação e comunicação 208, 230

Teleatendimento 202, 204

Telemedicina 133, 170, 230, 232

Telessaúde 230, 236

Tempos pandêmicos 271

Terapêutica multidisciplinar 190

Teste de schirmer 250, 255

Testes oftalmológicos 250

Transmissão de informações 271, 279

Transtorno de ansiedade generalizada 190, 192

Tuberculose (tb) 151,152

$\mathbf{U}$

Unidade de saúde da família (usf) 52, 55

Uso das máscaras 43, 45, 48 
Vacina 28, 31, 32, 33, 34, 37, 39, 40, 70, 72, 159, 161, 183, 184, 186, 221

Variantes $28,30,31,34,35,38,49,78$

Vídeos educativos 215, 226

Violência contra a mulher $163,169,170,173,174$

$\mathbf{X}$

Xeroftalmia 250,251 


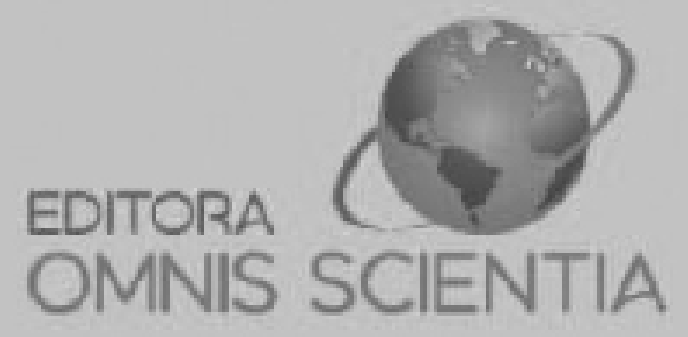

editoraomnisscientia@gmail.com M https://editoraomnisscientia.com.br/ @editora_omnis_scientia (0) https://wwww.facebook.com/omnis.scientia.9 $\boldsymbol{\oplus}$ +55 (87) 9656-3565 (C) 


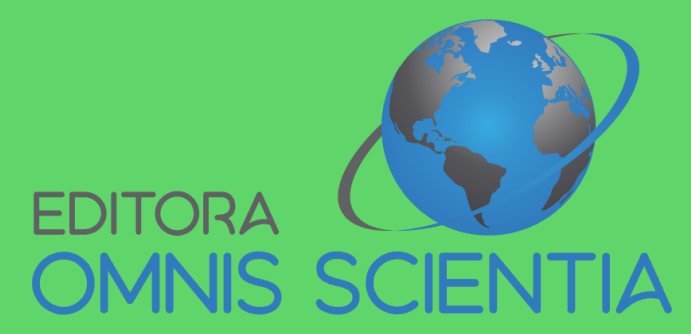

editoraomnisscientia@gmail.com M https://editoraomnisscientia.com.br/ (-) @editora_omnis_scientia (0) https://www.facebook.com/omnis.scientia.9 $\subsetneq$ +55 (87) 9656-3565 @ 